\title{
Reflets
}

Revue ontaroise d'intervention sociale et communautaire

\section{L'intervention sociale en français dans la région de Sudbury}

\section{Richard Carrière et Donald Dennie}

Volume 1, numéro 1, printemps 1995

Des pratiques à notre image : défis et ressources

URI : https://id.erudit.org/iderudit/026061ar

DOI : https://doi.org/10.7202/026061ar

Aller au sommaire du numéro

Éditeur(s)

Reflets : Revue ontaroise d'intervention sociale et communautaire

ISSN

1203-4576 (imprimé)

1712-8498 (numérique)

Découvrir la revue

Citer cet article

Carrière, R. \& Dennie, D. (1995). L'intervention sociale en français dans la région de Sudbury. Reflets, 1(1), 182-190. https://doi.org/10.7202/026061ar

Tous droits réservés (C) Reflets : Revue ontaroise d'intervention sociale et communautaire, 1995
Ce document est protégé par la loi sur le droit d'auteur. L'utilisation des services d'Érudit (y compris la reproduction) est assujettie à sa politique d'utilisation que vous pouvez consulter en ligne.

https://apropos.erudit.org/fr/usagers/politique-dutilisation/ 


\title{
L'intervention sociale en français dans la région de Sudbury
}

\author{
Richard Carrière \\ École de service social, Université Laurentienne \\ Donald Dennie \\ Département de sociologie, Université Laurentienne
}

\section{Introduction}

Depuis l'entrée en vigueur de la Loi sur les services en français (communément connue comme la Loi 8) en novembre 1989, le ministère des Services sociaux et communautaires cherche à améliorer l'accès aux services sociaux et communautaires en Ontario pour les citoyennes et citoyens francophones. Pour faciliter l'atteinte de cet objectif, des comités consultatifs sur les services en français ont été créés afin d'aviser les bureaux régionaux du ministère quant aux besoins de la communauté francophone.

Dans le cadre de son mandat, le Comité consultatif des services francophones de la région de Sudbury a choisi d'interroger les intervenantes et les intervenants qui travaillent en français au sujet de l'accessibilité et de l'utilisation du français dans leurs lieux de travail. Plus précisément, le comité cherchait à évaluer le niveau d'aise des intervenantes et intervenants face à l'utilisation du français, leur degré de satisfaction à l'égard de leur travail en français, leurs besoins liés à l'expression linguistique ainsi que les obstacles aux services en français.

C'est dans ce contexte que le Comité consultatif a demandé à l'Institut franco-ontarien de mener, à l'été 1993, une étude exploratoire. 


\section{Méthodologie}

Comme le Comité n'était pas en mesure de faire des entrevues individuelles, un questionnaire de six pages fut développé et expédié aux intervenantes et intervenants pouvant s'exprimer en français. Ce questionnaire contenait 12 questions fermées et trois questions ouvertes.

L'échantillonnage est non-probabiliste car afin d'identifier les sujets de l'étude, tous les responsables des organismes recevant des transferts de paiement de la part du ministère des Services sociaux et communautaires ainsi que d'autres organismes connexes ont été rejoints et invités à soumettre le nom de leur personnel pouvant s'exprimer en français. Nous avons demandé aux responsables des organismes qui n'ont pas voulu dévoiler l'identité de leur personnel de nous indiquer le nombre de personnes pouvant s'exprimer en français et de remettre euxmêmes le nombre correspondant de questionnaires et d'enveloppes pré-affranchies aux personnes concernées.

L'approche utilisée pour repérer les intervenantes et les intervenants pouvant s'exprimer en français ne garantit pas une identification complète. D'abord, le questionnaire n'a pas été distribué à toutes les personnes oeuvrant dans chaque organisme mais seulement à celles identifiées par l'administration. Nous ignorons de quelle façon les gestionnaires ont sélectionné les participantes et participants. Il est concevable que certaines personnes n'aient jamais dévoilé à la direction de l'agence le fait qu'elles parlent français, surtout dans des organismes où le français est très peu utilisé. De plus, plusieurs personnes ont choisi de ne pas répondre au questionnaire.

\section{Résultats}

Des 660 questionnaires distribués, 382 ont été complétés, ce qui correspond à un taux de participation de 57,8\% des intervenantes et intervenants identifiés comme pouvant s'exprimer en français dans les services sociaux et communautaires de la région de Sudbury. 
Reflets

\section{La communication avec la clientèle}

La plupart des 382 répondantes et répondants (70,7 \%) communiquent régulièrement en français avec la clientèle d'expression française; 137 disent communiquer souvent avec elle et 133 le font toujours. Rares sont les personnes qui refusent de le faire: seulement 5 ont répondu ne jamais s'exprimer en français. Dans presque la moitié des situations $(48,2 \%)$, ce sont les intervenantes et les intervenants qui initient la communication en français avec leurs clients tandis que dans 30,6\% des cas, c'est la clientèle qui prend cette initiative. Par ailleurs, lorsque vient le temps de rédiger les rapports au sujet de leur clientèle francophone, la majorité $(57 \%)$ le fait en anglais.

\section{La langue de communication dans les milieux de travail}

La langue de communication avec la direction et les cadres supérieurs est principalement l'anglais. La majorité des intervenantes et des intervenants ne communiquent jamais en français avec la direction $(54,5 \%)$ ou en supervision $(51,8 \%)$. Les communications avec le personnel de soutien et les collègues de travail se font assez fréquemment en français: $41,9 \%$ disent communiquer souvent ou toujours en français avec le personnel de soutien et presque la moitié $(48,4 \%)$ utilisent souvent ou toujours la langue française avec leurs collègues. Quant aux communications avec les autres organismes, les réponses sont partagées; les échanges en français se font souvent pour $32,2 \%$ des répondants et parfois pour un autre $32,2 \%$.

\section{Les personnes interrogées sont-elles à l'aise en français?}

La majorité, soit 246 des 382 sujets, jugent la qualité de leur français comme étant soit très bonne $(18,6 \%)$ ou bonne $(45,8 \%)$; $26,2 \%$ disent que leur français est plus ou moins bon, alors que $8,6 \%$ disent s'exprimer avec difficulté dans cette langue. 


\section{Le niveau de satisfaction avec les services en français}

Qu'en est-il de leur niveau de satisfaction avec les services en français dans leur propre agence? Une faible majorité (55,5\%) se dit très satisfaite ou satisfaite; $27,2 \%$ sont plus ou moins satisfaits, et $15,7 \%$ sont insatisfaits.

\section{Les obstacles au travail en français}

Les deux obstacles les plus fréquemment cités comme les empêchant de travailler en français sont:

- le milieu de travail est anglais (48,1\%)

- leur niveau d'expression en français est faible (16,3 \%).

L'étude démontre que les intervenantes et les intervenants ont accès à peu de ressources en français, notamment pour ce qui a trait aux conférences $(89,5 \%)$, aux ateliers de formation $(84,8 \%)$ et à la supervision $(64,4 \%)$.

Les formulaires $(41,8 \%)$ constituent la ressource la plus accessible et disponible en français.

\section{Les facteurs qui faciliteraient les communications en français}

Quels sont les facteurs qui permettraient de travailler davantage en français? Plusieurs personnes (17,9\%) identifient l'utilisation de documents, de manuels et de formulaires bilingues tandis que $16,5 \%$ suggèrent des cours de perfectionnement.

Questionnée quant à l'intérêt d'avoir accès à la traduction simultanée lors de conférences présentées en anglais, la très grande majorité $(80,1 \%)$ refuse cette option. Par contre, 48,4\% des répondantes et des répondants seraient intéressés à la possibilité de participer à des groupes de discussion en français lors de conférences présentées en anglais. Lorqu'on leur demande s'ils préfereraient des conférences en français, seulement 33,8\% ont répondu dans l'affirmative. 
Reflets

\section{Intérêt pour un regroupement}

Quoique $69 \%$ des répondantes et répondants seraient intéressés à rencontrer de temps à autre les intervenantes et intervenants des autres agences s'exprimant en français, la majorité (53,1\%) des répondantes ne sont pas intéressées à se joindre à un regroupement d'intervenantes et d'intervenants francophones.

\section{Intérêt pour un guide de services en français}

La grande majorité reconnait l'utilité d'un guide de services en français; 79,1\% le trouveraient utile pour eux-mêmes, 75,7 \% croient qu'il serait utile pour leurs collègues et $86,9 \%$ affirment qu'il le serait pour la clientèle. Seulement 55,2\% des répondantes et répondants aimeraient cependant que leur nom soit publié dans un tel guide.

\section{Discussion}

L'objectif principal de la loi 8 est d'assurer que la clientèle francophone des services gouvernementaux ait accès aux services en français. Cette étude nous dévoile que dans les organismes ayant des intervenantes et intervenants capables de s'exprimer en français, la clientèle est desservie régulièrement en français; 270 des 382 répondantes disent soit intervenir souvent ou toujours en français avec la clientèle. Par contre, un pourcentage important d'entres eux, soit $30 \%$, choisit de ne pas communiquer en français la plupart du temps, limitant ainsi l'accès aux services en français pour la clientèle francophone. En examinant de plus près les réponses obtenues, nous notons que ce sont principalement celles qui maitrisent bien ou très bien le français qui sont les plus aptes à communiquer en français.

Plus les intervenantes et les intervenants maitrisent le français, plus ils sont prêts à initier la communication avec la clientèle 
francophone. Quoique plusieurs (48,2 \%) disent initier la communication en français avec la clientèle francophone, leur rappelant ainsi leur droit de recevoir les services en français, un tiers laisse plutôt la clientèle faire le premier pas. Le fait de maîtriser le français ne garantit pas nécessairement que les intervenantes et les intervenants choisiront d'initier la communication en français, car à peu près un quart d'entre elles attendent que la clientèle s'exprime d'abord en français. Quel message donne-t-on à la clientèle francophone lorsqu'on adopte une telle attitude? Puisque moins de $50 \%$ des intervenantes et des intervenants affirment initier la communication en français, il serait important d'examiner, lors de futures consultations, les facteurs qui influencent le choix de la langue d'intervention.

Le contexte de travail constitue un facteur pouvant influencer ce choix. L'étude vient confirmer que l'anglais demeure la langue de communication dans la majorité des organismes de services sociaux et communautaires. Il est important de noter que très peu des organismes recevant des transferts de paiements du ministère des Services sociaux et communautaires dans la région sont désignés conformément à la Loi 8 sur les services en français. La communication avec la direction, les superviseurs et les collègues se fait principalement dans la langue de Shakespeare. Même les dossiers de la clientèle francophone sont rédigés en anglais. Pourtant, ces personnes ont le droit d'accès à leur dossier. L'étude démontre que ce sont les intervenantes et les intervenants qui maîtrisent le français qui sont les plus aptes à rédiger les dossiers en français. Par contre, nous notons aussi qu'un pourcentage important de ceux qui s'expriment très bien (40,8 \%) et bien $(55,4 \%)$ en français ne le font pas. Pourquoi? Les agences leur imposent-elles des restrictions d'expression linguistique?

Quoique les milieux de travail soient majoritairement anglophones, 55,5\% des intervenantes et des intervenants se sentent néanmoins très satisfaits $(18,3 \%)$ ou satisfaits $(37,2 \%)$ des services offerts en français dans leur boite; par contre, $44,5 \%$ ne le sont pas.

Une question ouverte invitait les personnes à se prononcer sur les obstacles qui les empêchent de travailler en français. Même si seulement $42 \%$ de l'échantillonnage a répondu à cette question, 
Reflets

une quarantaine de différentes réponses ont été exprimées. L'obstacle le plus présent provient du fait que le milieu de travail soit anglais $(48,1 \%)$. Par contre, seulement deux personnes ont spécifié que le français n'est pas accepté par l'administration. Ceci nous porte à suggérer que l'utilisation du français n'est pas formellement découragée, mais plutôt que l'infrastructure des organismes crée une situation où le français n'est tout simplement pas privilégié. Dans la majorité des cas, les cadres et les collègues de travail sont majoritairement anglophones et les documents et rapports sont en anglais.

Le deuxième obstacle le plus fréquemment cité est celui relié à la confiance des intervenantes et des intervenants quant à la qualité de leur français. En effet, 16,3\% d'entre eux ne se sentent même pas suffisamment à l'aise pour s'exprimer en français.

Lorsqu'on leur a demandé ce qui faciliterait l'utilisation du français, $28 \%$ désiraient avoir accès à des cours de perfectionnement et presque $20 \%$ suggéraient des ressources de travail en français tels des documents, des rapports et des formulaires. Afin de favoriser un milieu de travail en français, un petit groupe d'intervenantes $(9,6 \%)$ proposait même la création d'unités de travail francophones.

La pénurie de ressources en français vient rappeler le caractère anglophone des milieux de travail dans les organismes des services sociaux et communautaires de notre région. Les intervenantes et intervenants qui ont suggéré les ressources qu'elles aimeraient utiliser ont identifié en priorité les ateliers de formation en français $(25,7 \%)$ et les documents et manuels en français $(19,4 \%)$. Soulignons que moins de $10 \%$ ont demandé des conférences en français.

Les conférences sont tout de même l'une des principales activités d'éducation permanente pour les intervenantes et les intervenants dans le domaine des services sociaux et communautaires. Dans la très grande majorité des cas, elles sont offertes exclusivement en anglais. L'intérêt pour les conférences en français se limite aux personnes qui maîtrisent très bien cette langue. Nous ne savons pas pourquoi les autres ne s'y intéressent pas. 
Si les intervenantes et intervenants rejettent l'option des conférences en français, veulent-ils alors un service de traduction simultanée? En très grande majorité, cette option est également rejetée. Par contre, il y a un intérêt, de la part des personnes qui se sentent à l'aise en français, pour des groupes francophones de discussion lors des conférences présentées en anglais.

Quoique $62 \%$ des intervenantes et des intervenants apprécieraient se rencontrer de temps à autre avec leurs collègues des autres agences, cet intérêt ne se traduit pas par le désir de faire partie d'un réseau d'intervenantes et d'intervenants francophones. Il se peut que la formulation de la question ait détourné certaines personnes car nous leur avons demandé si elles seraient intéressées à faire partie d'un réseau francophone. Dans toutes les autres questions, nous n'avions pas spécifié le terme francophone. Autre que le désintérêt possible à se joindre à n'importe quel réseau, il se peut que la connotation d'affiliation culturelle ait influencé les personnes consultées. Ceux qui maîtrisent leur français s'intéressaient à l'idée d'un réseau. D'autre part, il semble que certains intervenantes et intervenants ne veulent pas dévoiler publiquement leur capacité de s'exprimer en français. Lorsque nous leur avons demandé si elles accepteraient que leur nom soit publié dans un guide de service en français, un pourcentage important, soit 41,1\%, a répondu non. Ce refus de dévoiler publiquement sa capacité d'expression française provient principalement des gens qui ne se sentent pas à l'aise avec leur français.

Quoique plusieurs ne veulent pas se faire identifier personnellement dans un répertoire, la majorité appuie la suggestion de créer un guide des services disponibles en français dans notre région.

\section{Conclusion}

Cette étude nous aide à mieux apprécier différents facteurs reliés à l'utilisation du français dans les services sociaux et communautaires en français dans notre région. En consultant les 
Reflets

intervenantes et les intervenants, nous commençons à mieux comprendre la complexité de l'accessibilité des services en français.

Cet accès implique beaucoup plus qu'une simple question d'embaucher des gens qui déclarent pouvoir s'exprimer en français. L'étude nous dévoile que la qualité de l'expression linguistique est importante car plus une personne est à l'aise en français, plus elle est apte à s'exprimer en français avec la clientèle francophone et plus grandes seront les chances qu'elle initie la communication en français.

Il nous semble important de retenir le contexte de pouvoir qui existe dans la relation entre la clientèle et les intervenants et les intervenants. Plusieurs personnes font des demandes de services dans une situation de crise. Elles n'osent souvent rien faire pour diminuer leur chance d'obtenir rapidement le service nécessaire. Ainsi, attendre que la clientèle choisisse de s'exprimer la première en français, lui impose une contrainte. Quand on tient compte du caractère anglophone dominant de plusieurs des services sociaux et communautaires de la région, on peut apprécier l'hésitation de la clientèle de s'exprimer d'abord en français, sinon d'insister pour se faire servir dans cette langue.

Le caractère anglophone de la majorité de nos organismes n'impose pas uniquement des barrières à la clientèle mais aussi aux intervenantes et aux intervenants qui se sentent peu outillés pour travailler en français. Plusieurs ont exprimé qu'il y a peu d'accès à des ressources en français et que leur travail en français n'est pas encadré. La supervision se fait surtout en anglais et le peu d'occasions de s'exprimer en français dans leur milieu de travail ne les aide pas à améliorer leur langue. Plusieurs sont insatisfaits des services en français dans leur agence. Le ministère des Services sociaux et communautaires, de concert avec les organismes sociaux et communautaires, doit trouver les stratégies qui encourageront vraiment les intervenantes et les intervenants à desservir la clientèle francophone en français. Elle y a droit! 